\title{
A Case of Intractable Hiccup Treated by Acupuncture and Moxibustion
}

\author{
Hui Sun'1, Yufeng Bi²*, Changchun Ji², Qinlang Liu' ${ }^{1}$ \\ 1Shaanxi University of Chinese Medicine, Xianyang 712046, Shaanxi Province, China \\ 2Shaanxi Provincial Hospital of Chinese Medicine, Xi'an 710003, Shaanxi Province, China \\ *Corresponding author: Yufeng Bi, byf111111@126.com
}

\begin{abstract}
Hiccup is a syndrome characterized by continuous sound in the throat, short and frequent sound, and difficult to stop ${ }^{[1]}$. Although the disease is not life-threatening, it seriously affects the quality of life of patients. Long term intractable hiccup will also have an impact on the psychology of patients and bring great trouble to patients. Hiccup is equivalent to diaphragmatic spasm in western medicine and involves a variety of systemic diseases ${ }^{[2]}$. At present, the treatment of this disease includes drugs and nerve block, but the curative effect and prognosis are not ideal. Acupuncture and moxibustion, as a green and simple treatment method, has unique curative effect in the treatment of hiccup. Now the test of one case of intractable hiccup caused by ineffective treatment is as follows.
\end{abstract}

Keywords: Hiccup; Acupuncture and moxibustion therapy; Case verification

Publication date: November 2021; Online publication: November 30, 2021

The patient, male, 65 years old, was treated for hiccups and vomiting for 1 month on September 14, 2019. One month ago, the patient suddenly developed a persistent cough without obvious cause, accompanied by chest tightness but he did not pay attention to it. During this period, Hiccups occurred intermittently. He was admitted to another hospital because he still made a grunting noise in his throat and he could not sleep at night. There were no obvious abnormalities in chest and abdomen CT and gastroscopy. He was diagnosed as "diaphragmatic spasm." After drug treatment (details unknown), the symptoms did not improve significantly, so he came to see a doctor.

There was a hum in his throat, which may be interpreted as a symptom. He hiccups every 4 to 5 seconds, is out of breath, and continues to hiccup. He has a low appetite, is melancholy, and has a grey and nervous appearance, as well as shortness of breath and weariness, chest and flank swelling and discomfort, inability to sleep at night, a pale tongue, white fur, and a weak pulse. Diaphragmatic spasm was the Western medicine diagnosis. Hiccup, spleen insufficiency, and kidney Yang were the TCM diagnoses. Tranquilizing and calming the nerves, balancing the stomach, lowering tension, warming and tonifying the spleen and kidneys were all part of the treatment. Acupuncture points was: Tousanshen (Benshen, Shenting), Sishencong, Taiyang, Cuanzhu, Yintang, Danzhong, Zhongwan, Qihai, Neiguan, Zusali, Sanyinjiao, Taichong, etc.

Operation methods:

(1) The patient took the supine position, exposed the skin of abdomen and lower limbs, and after routine disinfection with $75 \%$ alcohol at the local acupoints, use $0.25 \mathrm{~mm} \times 25 \mathrm{~mm}$ filiform needle in acupuncture to oblique Cuanzhu; Then select $0.25 \mathrm{~mm} \times 40 \mathrm{~mm}$ filiform needle in acupuncture to oblique Sishencong, Tousanshen, Yintang and Danzhong for 5 to $8 \mathrm{~mm}$, directly stab bilateral Taiyang, Neiguan and other acupoints of bilateral lower limbs for 5 to $8 \mathrm{~mm}$, and obliquely stab 
Qihai and Zhongwan for 10 to $13 \mathrm{~mm}$. Connect of bilateral Benshen with the positive and negative poles electroacupuncture therapeutic instrument, select continuous wave, frequency $40-60 \mathrm{hz}$, and take the patient's tolerance as the degree; The method of twisting stimulation is adopted for Cuanzhu. After the needle is entering and aerated, the twisting needle is strongly stimulated once every $5 \mathrm{~min}$. The rest of the acupoints were treated with neutral supplementation and draining method. The acupuncture was manipulated once in 15 minutes, taking the patient's tolerance to the acupuncture feeling as the degree. The acupuncture was casted on slowly after 30 minutes.

(2) Moxibustion and box moxibustion is used in the stomach duct region. Six small sections of 3 to $4 \mathrm{~cm}$ moxa sticks are respectively inserted into the holes, ignited, covered and placed on the stomach duct region. It can be adjusted at any time to ensure appropriate temperature. The moxibustion time is the same as the needle retention time.

(3) Auricular point sticking and pressing: Take Wangbuliuxing seed and stick it on the Menshen, Jiaogan, Erzhong, Wei, Benmen of both ears. Ask the patient to press it 3 to 4 times a day until the ears are red and hot.

(4) Combined with startle method, inhalation and breath holding method. After this treatment, the patient still had hiccups, the frequency decreased slightly, and the discomfort of chest and flank swelling was significantly relieved. Ask the patient to relax and come back the next day. The next morning, the patient came to the clinic to complain of hiccup, suddenly stopped, and had a good rest at night. Ask the patient to continue moxibustion to consolidate the curative effect after returning home, and pay attention to avoiding cold and keeping warm, eating and sleeping regularly. After 2 days of follow-up, the patient complained of no recurrence.

Traditional Chinese medicine believes that hiccups is caused by feeling external pathogens, improper diet, disharmony of emotions, deficiency of healthy Qi and injury to the middle energizer, resulting in gastric disharmony and depression and stomach Qi inversion. The disease is located in the diaphragm, and the diseased viscera are mainly in the stomach, involving the liver, spleen and kidney. The basic pathogenesis is gastric disharmony and depression and Qi inversion moving the diaphragm ${ }^{[1]}$, which is a common disease and syndrome in the spleen and stomach department of traditional Chinese medicine. Combined with the patient's hiccup and fear of cold, the method of "regulating Qi, reducing inversion, warming the middle and harmonizing the stomach" should be treated. Shenting, Dumai, Zutaiyang and Yangming, can calm the mind, awaken the brain and extinguish the wind; Benshen point is 3 inches next to the Shenting. It is the meeting of Zushaoyang and Yangwei. It has the effects of purging liver fire, clearing the head and calming the mind; Sishencong is a strange acupoint outside the meridians. It has the function of calming the mind, clearing the leader, refreshing the brain and opening the mind. The upper acupoints cooperate with the needling to calm the mind, regulate the mind and stop hiccups. Cuanzhu is the bladder meridian of the foot sun, which is located at the root of the eyebrow, close to the governor meridian, and is also the collateral of the governor meridian. It is the residue of Qi and blood. Acupuncture at this acupoint can promote Qi and promote blood circulation to remove meridian obstruction, and Qi circulation leads to blood circulation, so it can guide Qi downward and stop hiccups; Danzhong is the "Qi meeting" of Bahui point. Acupuncture at this point can straighten out the Qi mechanism of triple energizers, broaden the chest and reduce inversion, and be good at treating chest and diaphragm diseases. Zhongwan is the $\mathrm{Mu}$ front acupoints and influential point of Fu-organs of the stomach, which can strengthen the movement of the middle energizer, dredge and lower the Fu Qi, and be good at treating spleen and stomach diseases; Zusanli is the Xiahe point under the stomach, Sanyinjiao is the intersection point of the three meridians of liver, spleen and kidney, which can strengthen the spleen and calm the mind. The combination of the three can strengthen the spleen and stomach inverse. The sea of Qi is the sea where original Qi originates. It can replenish Qi and help Yang, promote Qi and dissipate stagnation. The book "Shou Shi Bao Yuan" says: 
"Treating hiccups should take routine moxibustion on Qihai"; Neiguan point is connected with Yinwei Meridian, and is the collateral point of hand Jueyin pericardium meridian, which can not only smooth the Qi of chest and diaphragm, but also unblock the triple energizers Qi machine and calm the heart and stomach; Taichong is the Yuan-source point and Shu point of liver meridian, which is also to regulate Qi and calm the adverse-rising energy; With the cooperation of various acupoints, if the Qi is smooth, the hiccup stops, and if the Qi is sufficient, the pulse is also full, so the hiccup stops. In the selected auricular points, Shenmen can improve sleep and neurasthenia, Jiaogan can correct gastrointestinal and autonomic nerve dysfunction, Erzhong can treat hiccup, Weidian can relieve gastric spasm, and Benmen can improve cardiac spasm. The combination of various points can dredge meridians, dredge collaterals, smooth Qi and regulate viscera. Mild moxibustion in the stomach duct region is called "warming the middle and harmonizing the stomach." Acupuncture and moxibustion combined with auricular point sticking and pressing can play a synergistic therapeutic role, so it has a significant effect on hiccup.

When looking at the etiology from a Western medical standpoint, the occurrence of a hiccup requires a reflex arc that includes the afferent nerve, central nerve, and efferent nerve. Hiccups can be caused by any factor that affects any link of the reflex arc ${ }^{[3]}$. Acupuncture and moxibustion may influence the hiccup reflex, resulting in a hiccup-stopping effect. Plain Questions, Treatise on Cough: "All the internal organs, not just the lung, cause people to cough," as does hiccup. In the clinic, numerous reasons of hiccup can be seen, but the pathogenesis of traditional Chinese medicine is always one, which is produced by a malfunction of the Qi mechanism. As a result, in acupuncture and moxibustion clinical therapy, we should understand the pathophysiology of hiccup, specifically evaluate which viscera lead to Qi mechanism disturbance, and then dialectically pick points based on the specific meridians of the afflicted viscera organs. To improve the clinical curative impact, we must first comprehend its theory before selecting points in clinical syndrome.

\section{Disclosure statement}

The authors declare no conflict of interest.

\section{References}

[1] Liang F, Wang H, 2016, Acupuncture and Moxibustion. China Press of Traditional Chinese Medicine, Beijing, 236.

[2] Tang G, 2012, Research Progress in Etiology, Pathogenesis and Treatment of Hiccup, ACTA Chinese Medicine, 27(7): 878-879.

[3] Xu J, Dai, Ge J, et al., 2017, Research Progress in Pathogenesis, Diagnosis and Treatment Strategies of Hiccup. Chinese Journal of Medicine, 52(06): 17-20. 